\title{
How to Recover Any Byte of Plaintext on RC4
}

\author{
Toshihiro Ohigashi ${ }^{1(凶)}$, Takanori Isobe ${ }^{2}$, Yuhei Watanabe ${ }^{2}$, \\ and Masakatu Morii ${ }^{2}$ \\ 1 Hiroshima University, 1-4-2 Kagamiyama, \\ Higashi-Hiroshima, Hiroshima 739-8511, Japan \\ ohigashi@hiroshima-u.ac.jp \\ 2 Kobe University, 1-1 Rokkoudai, Nada-ku, Kobe 657-8501, Japan \\ Takanori.Isobe@jp. sony.com \\ yuheiwatanabe@stu.kobe-u.ac.jp \\ mmorii@kobe-u.ac.jp
}

\begin{abstract}
In FSE 2013, Isobe et al. proposed efficient plaintext recovery attacks on RC4 in the broadcast setting where the same plaintext is encrypted with different user keys. Their attack is able to recover first 1000 terabytes of a plaintext with probability of almost one, given $2^{34}$ ciphertexts encrypted by different keys. Since their attack essentially exploits biases in the initial (1st to 257th) bytes of the keystream, it does not work any more if such initial bytes are disregarded. This paper proposes two advanced plaintext recovery attacks that can recover any byte of a plaintext without relying on initial biases, i.e., our attacks are feasible even if initial bytes of the keystream are disregarded. The first attack is the modified Isobe et al.'s attack. Using the partial knowledge of the target plaintext, e.g., only 6 bytes of the plaintext, the other bytes can be recovered with the high probability from $2^{34}$ ciphertexts. The second attack does not require any previous knowledge of a plaintext. In order to achieve it, we develop a guess-and-determine plaintext recovery method based on two strong long-term biases. Given $2^{35}$ ciphertexts, any byte of a plaintext can be recovered with probability close to one.
\end{abstract}

Keywords: RC4 $\cdot$ Broadcast setting $\cdot$ Plaintext recovery attack $\cdot$ Bias · Guess-and-determine attack $\cdot$ Multi-session setting $\cdot$ RC4-drop

\section{Introduction}

RC4, designed by Rivest in 1987, is one of most widely used stream ciphers in the world. It is adopted in many software applications and standard protocols such as SSL/TLS, WEP, Microsoft Lotus and Oracle secure SQL. RC4 consists of a key scheduling algorithm (KSA) and a pseudo-random generation algorithm (PRGA). The KSA converts a user-provided variable-length key (typically, 5-32 bytes) into an initial state $S$ consisting of a permutation of $\{0,1,2, \ldots, N-1\}$, where $N$ is typically 256 . The PRGA generates a keystream $Z_{1}, Z_{2}, \ldots, Z_{r}$, $\ldots$ from $S$, where $r$ is a round number of the PRGA. $Z_{r}$ is XOR-ed with the 
$r$-th plaintext byte $P_{r}$ to obtain the ciphertext byte $C_{r}$. The algorithm of RC4 is shown in Algorithm 1, where + denotes arithmetic addition modulo $N, \ell$ is the key length, and $i$ and $j$ are used to point to the locations of $S$, respectively. Then, $S[x]$ denotes the value of $S$ indexed $x$.

In FSE 2001, Mantin and Shamir proposed a plaintext recovery attack on RC4 in the broadcast setting where the same plaintext is encrypted with different user keys [12]. Using a bias of $Z_{2}$, a second byte of the plaintext is recovered from $\Omega(N)$ ciphertexts encrypted with randomly-chosen different keys. In FSE 2011, Maitra, Paul and Sen Gupta showed that $Z_{3}, Z_{4}, \ldots, Z_{255}$ are also biased to 0 [9]. The bytes 3 to 255 are also obtained in the broadcast setting, from $\Omega\left(N^{3}\right)$ ciphertexts. In FSE 2013, Isobe et al. introduced several new biases in the initial bytes of the RC4 keystream, and constructed a cumulative list of strong biases in the first 257 bytes with theoretical reasons [7]. They demonstrated plaintext recovery attacks using their strong biases set with typical parameters of $N=256$ and $\ell=16$ (128-bit key). $2^{32}$ ciphertexts encrypting the same plaintext enable to extract first 257 bytes of a plaintext with probability more than 0.8 . Using these initial biases in conjunction with the digraph repetition bias proposed by Mantin in EUROCRYPT 2005 [11], the consecutive first 1000 terabytes of a plaintext is theoretically recovered with probability of almost one from $2^{34}$ ciphertexts encrypted by different keys. After that, AlFardan et al. also proposed similar plaintext recovery attack of the first 256 bytes [1] independently of [7], and this attack can recover first 256 bytes of a plaintext with probability more than 0.96 from $2^{32}$ ciphertexts encrypted by different keys. Note that broadcast attacks $[1,7]$ can be converted into the attacks for the multi-session setting of SSL/TLS where the target plaintext blocks are repeatedly sent in the same position in the plaintexts in multiple sessions [3].

Previous plaintext recovery attacks essentially exploit biases in the 1st to 257 th bytes of the keystream. If the initial $256 / 512 / 768$ bytes of the keystream are disregarded, as recommended in case of RC4 usages, it does not work any more as mentioned in [7]. Thus, RC4 that disregards the first $n$ bytes of a keystream seem to be secure against above attacks for $n>257$.

This paper proposes two advanced plaintext recovery attacks that can recover any byte of a plaintext without relying on initial biases of the keystream, i.e., our attacks are feasible even if initial bytes of the keystream are disregarded, unlike Isobe et al. and AlFardan et al.'s attacks. To begin with, we improve Isobe et al.'s attack so that it works without initial biases of a keystream. In particular, we assume that an attacker knows some bytes of the target plaintext, e.g., fixed header information. Using the digraph repetition biases in forward and backward manners, the other bytes of the plaintext are recovered from the partial knowledge of the plaintext. In our attack, if only consecutive 6 bytes of the target plaintexts are known, 1000 terabytes of the target plaintext can be recovered with probability of about 0.636 from $2^{34}$ ciphertexts. The number of required ciphertexts of this attack is same as that of Isobe et al.'s attack, while Isobe et al.'s attack needs the initial 257 bytes of the keystream. The second attack does not require any previous knowledge of a plaintext. In order to achieve 


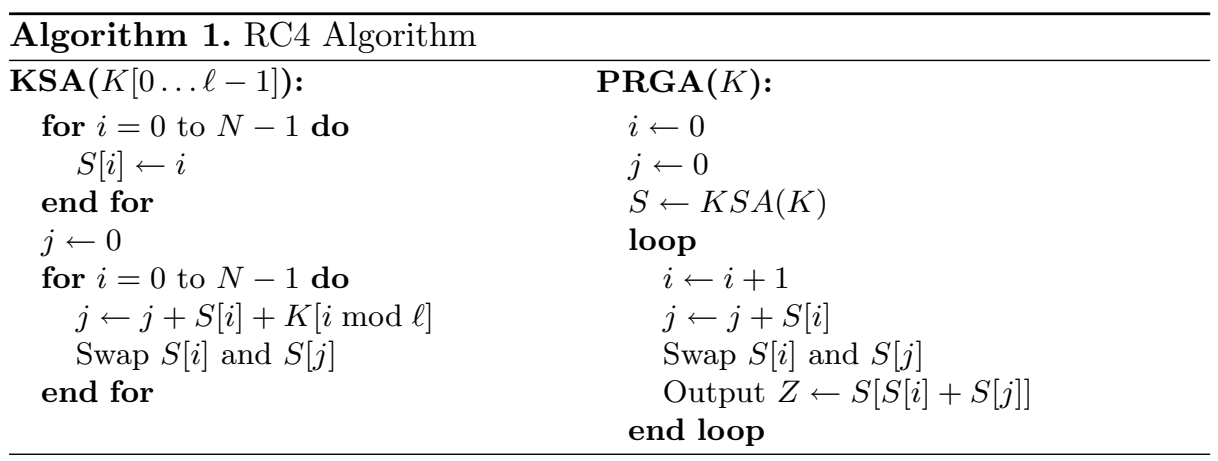

it, we develop a novel guess-and-determine plaintext recovery method based on two strong long-term biases, i.e., digraph repetition biases [11] and FluhrerMcGrew biases [4]. The basic idea behind our guess-and-determine attack is that two biases are used for the detection the wrong candidates of plaintext bytes. Given $2^{35}$ ciphertext encrypted by different keys, any byte of a plaintext can be recovered with probability close to one ${ }^{1}$.

We emphasize that our attacks are applicable even if any number of initial bytes of the keystream are disregarded, with almost same amount of ciphertexts as Isobe et al.'s attack. Therefore, our work reveals that the RC4 implementation that disregards the first $n$ bytes of a keystream is also not secure even if $n$ is enough large (e.g. $n=3072$ ).

\section{Preliminary}

In this section, we introduce two known long-term biases, which occur in any keystream bytes, because our attacks are based on them. Then we describe previous plaintext recovery attacks on RC4 in the broadcast setting.

\subsection{Long-term Bias}

As a long-term bias, following two types of biases were proposed.

Bias of Digraph Probabilities (FM00 Bias). Fluhrer and McGrew showed a long-term bias of digraph probabilities in the RC4 keystream, called the FMOO bias. It is a bias of 2-byte word of the keystream with the condition of index $i$ $(=r \bmod N)$ [4], and consists of 12 positive or negative events. The detail of the FM00 bias is shown in Table 1.

\footnotetext{
${ }^{1}$ Independently of our work, other plaintext recovery attacks on RC4 implementation which disregards the first $n$ bytes of a keystream, was recently reported in $[1,2]$. The attack uses only the Fluhrer-McGrew biases with the sophisticated count-up method, and obtains experimental results similar to that of our attack.
} 
Table 1. Events of the FM00 bias with the condition of index $i(=r \bmod N)$

\begin{tabular}{lll}
\hline Condition of event & Digraph $\left(Z_{r}, Z_{r+1}\right)$ & $\operatorname{Pr}\left(Z_{r} \wedge Z_{r+1}\right)$ \\
\hline$i=1$ & $(0,0)$ & $N^{-2} \cdot\left(1+2 \cdot N^{-1}\right)$ \\
$i \neq 1, N-1$ & $(0,0)$ & $N^{-2} \cdot\left(1+N^{-1}\right)$ \\
$i \neq 0,1$ & $(0,1)$ & $N^{-2} \cdot\left(1+N^{-1}\right)$ \\
$i \neq N-2$ & $(i+1, N-1)$ & $N^{-2} \cdot\left(1+N^{-1}\right)$ \\
$i \neq 1, N-2$ & $(N-1, i+1)$ & $N^{-2} \cdot\left(1+N^{-1}\right)$ \\
$i \neq 0, N-3, N-2, N-1$ & $(N-1, i+2)$ & $N^{-2} \cdot\left(1+N^{-1}\right)$ \\
$i=N-2$ & $(N-1,0)$ & $N^{-2} \cdot\left(1+N^{-1}\right)$ \\
$i=N-1$ & $(N-1,1)$ & $N^{-2} \cdot\left(1+N^{-1}\right)$ \\
$i=0,1$ & $(N-1,2)$ & $N^{-2} \cdot\left(1+N^{-1}\right)$ \\
$i=2$ & $(N / 2+1, N / 2+1)$ & $N^{-2} \cdot\left(1+N^{-1}\right)$ \\
$i \neq N-2$ & $(N-1, N-1)$ & $N^{-2} \cdot\left(1-N^{-1}\right)$ \\
$i \neq 0, N-1$ & $(0, i+1)$ & $N^{-2} \cdot\left(1-N^{-1}\right)$ \\
\hline
\end{tabular}

The Digraph Repetition Bias ( $\boldsymbol{A} \boldsymbol{B} \boldsymbol{S A B}$ Bias). Mantin found another longterm bias of digraph distribution in the RC4 keystream [11], called the $A B S A B$ bias. Assuming $A$ and $B$ are two words of the keystream, the digraph $A B$ tends to repeat with short gaps $S$ between them, e.g., $A B A B, A B C A B$ and $A B C D A B$, where gap $S$ is defined as zero, $C$, and $C D$, respectively. The detail of the $A B S A B$ bias is as follows,

$$
Z_{r}\left\|Z_{r+1}=Z_{r+2+G}\right\| Z_{r+3+G} \text { for } G \geq 0
$$

where $\|$ is a concatenation. The probability that Eq. (1) holds is given as Theorem 1.

Theorem 1 [11]. For small values of $G$ the probability of the pattern $A B S A B$ in RC4 keystream, where $S$ is a G-byte string, is $\left(1+e^{(-4-8 G) / N} / N\right) \cdot 1 / N^{2}$.

\subsection{Previous Works}

This section briefly reviews known attacks on RC4 in the broadcast setting where the same plaintext is encrypted with different randomly-chosen keys.

Mantin-Shamir (MS) Attack. Mantin and Shamir first presented broadcast RC4 attacks. Their attacks exploit a bias of second byte of keystream, $Z_{2}$ [12] as follows.

Theorem 2 [12]. Assume that the initial permutation $S$ is randomly chosen from the set of all the possible permutations of $\{0,1,2, \ldots, N-1\}$. Then the probability that the second output byte of $R C_{4}$ is 0 is approximately $\frac{2}{N}$.

This probability is estimated as $\frac{2}{256}$ when $N=256$. Based on this bias, a distinguishing attack and a plaintext recovery attack on $\mathrm{RC} 4$ in the broadcast setting are demonstrated by Theorems 3 and 4, respectively. 
Theorem 3 [12]. Let $X$ and $Y$ be two distributions, and suppose that the event $e$ happens in $X$ with probability $p$ and in $Y$ with probability $p \cdot(1+q)$. Then for small $p$ and $q, \mathrm{O}\left(\frac{1}{p \cdot q^{2}}\right)$ samples suffice to distinguish $X$ from $Y$ with a constant probability of success.

In this case, $p$ and $q$ are given as $p=1 / N$ and $q=1$. The number of samples is about $\frac{1}{p \cdot q^{2}}=N$.

Theorem 4 [12]. Let $P$ be a plaintext, and let $C^{(1)}, C^{(2)}, \ldots, C^{(k)}$ be the $R C 4$ encryptions of $P$ under $k$ uniformly distributed keys. Then, if $k=\Omega(N)$, the second byte of $P$ can be reliably extracted from $C^{(1)}, C^{(2)}, \ldots, C^{(k)}$.

According to the relation $C_{2}^{(i)}=P_{2}^{(i)} \oplus Z_{2}^{(i)}$, if $Z_{2}^{(i)}=0$ holds, then $C_{2}^{(i)}$ is same as $P_{2}^{(i)}$. From Theorem $2, Z_{2}=0$ occurs with twice the expected probability of a random one. Thus, most frequent byte in amongst $C_{2}^{(1)}, C_{2}^{(2)}, \ldots, C_{2}^{(k)}$ is likely to be $P_{2}$ itself. When $N=256$, it requires more than $2^{8}$ ciphertexts encrypted with randomly-chosen keys.

Maitra-Paul-Sen Gupta (MPS) Attack. Maitra, Paul and Sen Gupta showed that $Z_{3}, Z_{4}, \ldots, Z_{255}$ are also biased to $0[6,9]$. Although Mantin and Shamir assume that an initial permutation $S$ is random, Maitra et al. exploit biases of $S$ after the KSA [10]. Then the 3rd to 255th bytes of a plaintext are obtained from $\Omega\left(N^{3}\right)$ ciphertexts encrypted with different keys.

Isobe-Ohigashi-Watanabe-Morii (IOWM) Attack. Isobe et al. proposed a full plaintext recovery attack, which is able to extract the full bytes of a plaintext on RC4 from ciphertexts in the broadcast setting [7]. Their attack consists of two phases: an initial byte recovery phase and a sequential recovery phase for finding later bytes of a plaintext.

In the initial byte recovery phase, the first 257 bytes of a plaintext are recovered by using the cumulative bias set of $Z_{1}, Z_{2}, \ldots, Z_{257}$. Their cumulative bias set includes a conditional bias $Z_{1}=0 \mid Z_{2}=0$ [7] and single byte biases $Z_{2}=0$ [12], $Z_{3}=131$ [7], $Z_{r}=0$ for $3 \leq r \leq 255[6,9], Z_{r}=r$ for $3 \leq r \leq 255$ [7], $Z_{16}=240$ [5], $Z_{r}=(256-r)$ for $r=32,48,64,80,96,112$ [7], and $Z_{256} \neq 0[7], Z_{257}=0[7]$ (when $N=256$ and $\ell=16$ ). Given $2^{32}$ ciphertexts encrypted by randomly-chosen keys, the first 257 bytes of a plaintext are extracted with probability more than 0.8 .

In the sequential recovery phase, the later bytes (after $P_{258}$ ) are sequentially recovered with the first 257 bytes of the plaintext, which were already obtained in the initial byte recovery phase. The sequential algorithm effectively uses a longterm bias, the $A B S A B$ bias [11]. In particular, $A B S A B$ biases with different $G$ are simultaneously used for enhancing the attack, using the following lemmas for the discrimination.

Lemma 1 [11]. Let $X$ and $Y$ be two distributions and suppose that the independent events $\left\{e_{i}: 1 \leq i \leq k\right\}$ occur with probabilities $\operatorname{Pr}_{X}\left(e_{i}\right)=p_{i}$ in $X$ and 
$\operatorname{Pr}_{Y}\left(e_{i}\right)=\left(1+q_{i}\right) \cdot p_{i}$ in $Y$. Then the discrimination $D$ of the distributions is $\sum_{i} p_{i} \cdot q_{i}^{2}$.

The number of required samples for distinguishing the biased distribution from the random distribution with probability of $1-\alpha$ is given as the following lemma.

Lemma 2 [11]. The number of samples that is required for distinguishing two distributions that have discrimination $D$ with success rate $1-\alpha$ (for both directions) is $(1 / D) \cdot(1-2 \alpha) \cdot \log _{2} \frac{1-\alpha}{\alpha}$.

This lemma shows that in the broadcast RC4 attack, once the discrimination $D$ and the number of samples $k$ are given, the success probability $\operatorname{Pr}_{\text {distinguish }}$ for distinguishing the distribution of correct candidate plaintext byte (the biased distribution) from the distribution of one wrong candidate of plaintext byte (a random distribution) always becomes constant. The success probability for recovering plaintext bytes depends on $\operatorname{Pr}_{\text {distinguish }}$. Thus if $k$ is fixed, the success probability only depends on $D$.

In their attack, the following equation regarding the $A B S A B$ bias is used.

$$
\begin{aligned}
& \left(C_{r} \| C_{r+1}\right) \oplus\left(C_{r+2+G} \| C_{r+3+G}\right) \\
& =\left(P_{r} \oplus Z_{r} \| P_{r+1} \oplus Z_{r+1}\right) \oplus\left(P_{r+2+G} \oplus Z_{r+2+G} \| P_{r+3+G} \oplus Z_{r+3+G}\right) \\
& =\left(P_{r} \oplus P_{r+2+G} \oplus Z_{r} \oplus Z_{r+2+G} \| P_{r+1} \oplus P_{r+3+G} \oplus Z_{r+1} \oplus Z_{r+3+G}\right) .
\end{aligned}
$$

Assuming that Eq. (1) (event of the $A B S A B$ bias) holds, the relation of plaintexts and ciphertexts without keystreams is obtained, i.e., $\left(C_{r} \| C_{r+1}\right) \oplus\left(C_{r+2+G}\right.$ $\left.\| C_{r+3+G}\right)=\left(P_{r} \oplus P_{r+2+G} \| P_{r+1} \oplus P_{r+3+G}\right)=\left(P_{r} \| P_{r+1}\right) \oplus\left(P_{r+2+G} \| P_{r+3+G}\right)$. For combining these relations with different $G$ to enhance the biases, the algorithm uses the knowledge of pre-guessed plaintext bytes. For example, in the cases of $\left(r=r^{\prime}\right.$ and $\left.G=1\right)$ and $\left(r=r^{\prime}+1\right.$ and $\left.G=0\right)$, right parts of equations are given as $\left(P_{r^{\prime}} \| P_{r^{\prime}+1}\right) \oplus\left(P_{r^{\prime}+3} \| P_{r^{\prime}+4}\right)$ and $\left(P_{r^{\prime}+1} \| P_{r^{\prime}+2}\right) \oplus\left(P_{r^{\prime}+3} \| P_{r^{\prime}+4}\right)$, respectively. Then, if $P_{r^{\prime}}, P_{r^{\prime}+1}$, and $P_{r^{\prime}+2}$ are already known, the two equations with respected to $\left(P_{r^{\prime}+3} \| P_{r^{\prime}+4}\right)$ is obtained by transposing $P_{r^{\prime}}, P_{r^{\prime}+1}$, and $P_{r^{\prime}+2}$ to the left part of the equation. Then, these equations with different $G$ can be merged.

Suppose that $P_{1}, P_{2}, \ldots, P_{257}$ are guessed by the cumulative bias set. Then, the sequential algorithm for recovering $P_{r}$ for $r=258,259, \ldots, P_{M A X}$, from $k$ ciphertexts $C^{(1)}, C^{(2)}, \ldots, C^{(k)}$ encrypted by different keys, by using $A B S A B$ biases of $G=0,1, \ldots, G_{M A X}$ is given as follows.

Step 1 Obtain $C_{258-3-G_{M A X}}, C_{258-2-G_{M A X}}, \ldots, C_{P_{M A X}}$ in each ciphertext, and make frequency tables $T_{\text {count }}[r][G]$ of $\left(C_{r-3-G} \| C_{r-2-G}\right) \oplus\left(C_{r-1} \| C_{r}\right)$ for all $r=258,259, \ldots, P_{M A X}$ and $G=0,1, \ldots, G_{M A X}$, where $\left(C_{r-3-G} \|\right.$ $\left.C_{r-2-G}\right) \oplus\left(C_{r-1} \| C_{r}\right)=\left(P_{r-3-G} \| P_{r-2-G}\right) \oplus\left(P_{r-1} \| P_{r}\right)$ only if Eq. (1) holds.

Step 2 Set $r=258$.

Step 3 Guess the value of $P_{r}$. 
Step 3.1 For $G=0,1, \ldots, G_{M A X}$, convert $T_{\text {count }}[r][G]$ into a frequency table $T_{\text {marge }}[r]$ of $\left(P_{r-1} \| P_{r}\right)$ by using pre-guessed values of $P_{r-3-G_{M A X}}$, $\ldots, P_{r-2}$, and merge counter values of all tables.

Step 3.2 Make a frequency table $T_{\text {guess }}[r]$ indexed by only $P_{r}$ from $T_{\text {marge }}[r]$ with knowledge of the $P_{r-1}$. To put it more precisely, using a pre-guessed value of $P_{r-1}$, only tables $T_{\text {marge }}[r]$ corresponding to the value of $P_{r-1}$ is taken into consideration. Finally, regard most frequency one in table $T_{\text {guess }}[r]$ as the correct $P_{r}$.

Step 4 Increment $r$. If $r=P_{M A X}+1$, terminate this algorithm. Otherwise, go to Step 3.

Isobe et al. theoretically showed that this algorithm can recover consecutive 1000 terabytes of a plaintext from $2^{34}$ ciphertexts when $G_{M A X}=63(D=$ $\left.2^{-28.03}\right)$ is adopted.

Countermeasure. These attacks essentially exploit biases in the initial (1st to 257th) bytes of the RC4 keystream. If initial bytes of the keystream are disregarded, it does not work any more as mentioned in [7]. Thus, the RC4$\operatorname{drop}(257)$ is considered as a countermeasure against previous plaintext recovery attacks, where RC4- $\operatorname{drop}(n)$ is an RC4 implementation that disregards the first $n$ bytes of a keystream ${ }^{2}$.

In addition, Mironov also recommended $n=512$ or 768 , and gave a conservative recommended parameter $n=3072$ based on the experimental data for avoiding initial bytes biases [13].

\section{Plaintext Recovery Attack Using Known Partial Plaintext Bytes}

In this section, we propose a plaintext recovery attack that is feasible even if initial bytes of a keystream are disregarded, unlike previous attacks. We improve Isobe et al.'s attack so that it works without initial biases of the keystream. In particular, we suppose that an attacker has the partial knowledge of a target plaintext, e.g., fixed header information. This assumption is reasonable in the practical usage on $\mathrm{RC} 4$. Then, with partial knowledge of the target plaintext, the other bytes of the plaintext can be recovered by using $A B S A B$ biases.

For the simplification, the encryption on $\operatorname{RC} 4-\operatorname{drop}(n)$ denotes $C_{r}=P_{r} \oplus$ $Z_{r+n}=P_{r} \oplus Z_{r^{*}}$ where $r^{*}=r+n$.

\subsection{Attack Functions}

We give two functions based on $A B S A B$ biases for recovering an unknown byte of the plaintext.

\footnotetext{
$\overline{{ }^{2} \mathrm{RC} 4-\operatorname{drop}(n)}$ is a generalized implementation of the countermeasure written by [13],
} and this is defined at http://www.users.zetnet.co.uk/hopwood/crypto/scan/cs.html. 


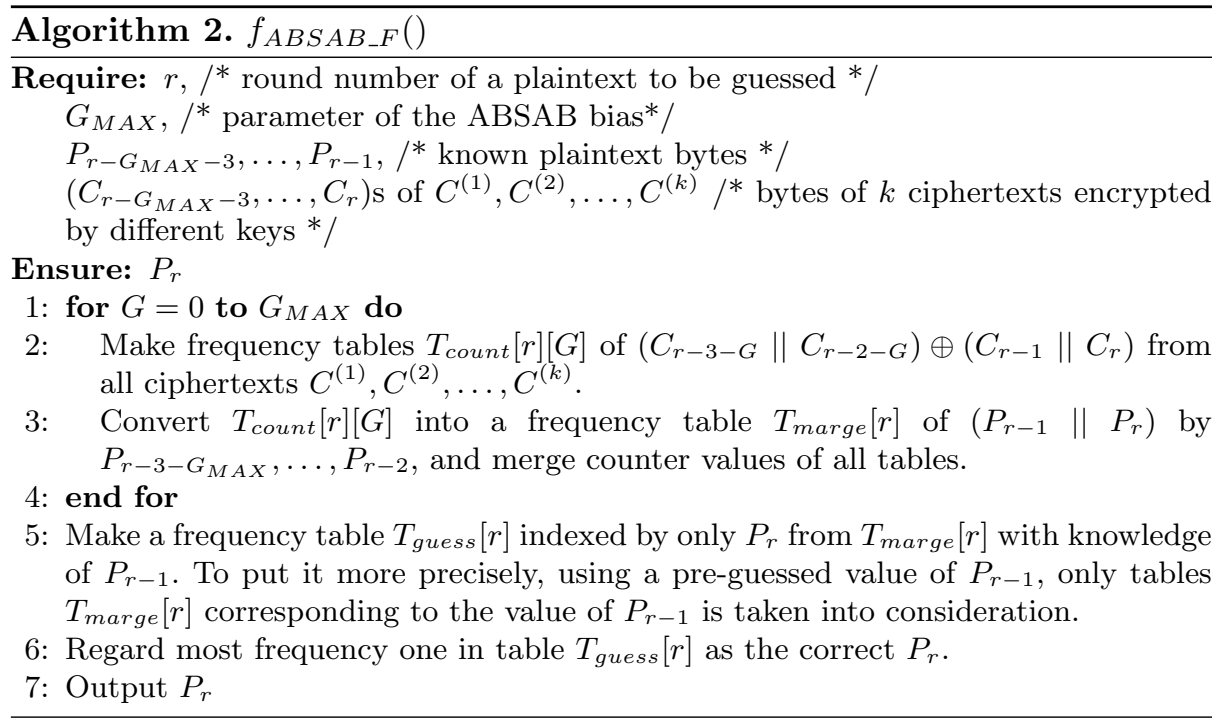

$f_{A B S A B_{-} F}()$ : Find an unknown byte $P_{r}$ from pre-known consecutive $\left(G_{M A X}+\right.$ 3 ) bytes of a plaintext $P_{r-G_{M A X}-3}, \ldots, P_{r-1}$ (See Algorithm 2).

$f_{A B S A B_{-} B}()$ : Find an unknown byte $P_{r}$ from pre-known consecutive $\left(G_{M A X}+\right.$

3) bytes of a plaintext $P_{r+1}, \ldots, P_{r+G_{M A X}+3}$.

The algorithm of $f_{A B S A B_{-} B}()$ is given by replacing "-" of subscripts of variables in Algorithm 2 to "+". These functions can be obtained from Step 1 and Step 3 of the IOWM attack. Figure 1 (Fig. 3) illustrates the procedures of $f_{A B S A B_{-} F}()$ and $f_{A B S A B_{-} B}()$. By using above two functions, all plaintext bytes can be recovered from the partial knowledge of the plaintext and the corresponding ciphertexts.

\subsection{Attack Procedure}

Suppose that $x$ bytes of a target plaintext, $P_{r}, \ldots P_{r+x-1}$, are given. An attacker aims to recover the next byte $\left(P_{r+x}\right)$ or the previous byte $\left(P_{r-1}\right)$ of the known plaintext bytes by using $f_{A B S A B_{-} F}()$ or $f_{A B S A B_{-} B}()$, respectively. If $\left(P_{r+x}\right)$ or $\left(P_{r-1}\right)$ is successfully recovered, the attacker recovers $\left(P_{r+x+1}\right)$ or $\left(P_{r-2}\right)$ with knowledge of $\left(P_{r+x}\right)$ or $\left(P_{r-1}\right)$. Since $G_{M A X}$ increases, the probability for recovering a plaintext byte also increases in the next step.

Our attack repeats above procedure until the all plaintext bytes are found. After $G_{M A X}$ reaches $63, G_{M A X}$ is fixed since the increase of $D$ is converged around $G_{M A X}=63$ as mentioned in [7]. Figure 2 shows that our plaintext recovery attack using known partial plaintext bytes when consecutive 6 bytes of a target plaintext are given. 


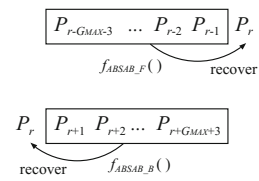

Fig. 1. Forward and backward functions for recovering one byte of a target plaintext using the partial knowledge of the plaintext and $A B S A B$ biases

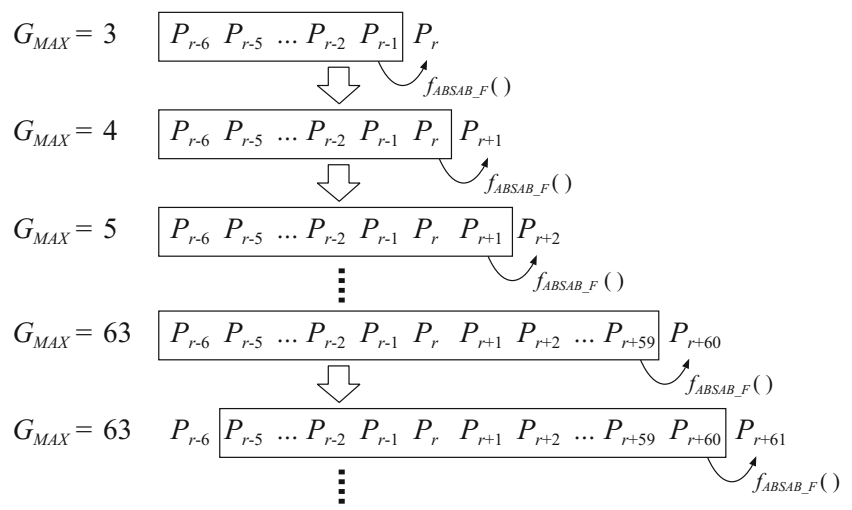

Fig. 2. A plaintext recovery attack using the known partial plaintext bytes when consecutive 6 bytes of a target plaintext are known

\subsection{Experimental Results}

We evaluate our plaintext recovery attack on $\operatorname{RC} 4-\operatorname{drop}(n)$ in the broadcast setting by the computer experiment when $N=256$ and $n=3072$, which is a conservative recommended parameter given in [13]. Then, ciphertext $C$ is expressed as $\left(C_{1}, C_{2}, \ldots, C_{r}, \ldots\right)=\left(P_{1} \oplus Z_{1+3072}, P_{2} \oplus Z_{2+3072}, \ldots, P_{r} \oplus Z_{r+3072}, \ldots\right)$.

In order to estimate the success probability of our attack, we evaluate the probabilities for recovering the one byte of the target plaintext by $f_{A B S A B_{-} F}()$ and $f_{A B S A B_{-} B}()$. The probabilities dependent on $G_{M A X}$ and the number of obtained ciphertexts, but does not depend on the round number $r$. Thus, our experiment uses parameters such that $G_{M A X}=0,1,2,3,4,5,6,7,8,9,15,31,63$ and $2^{31}, 2^{32}, \ldots, 2^{36}$ ciphertexts.

Table 2 shows the experimental result for 128 different plaintexts when $r=$ $128, n=3072$, and the number of known plaintext bytes and the discrimination $D$ and corresponding $G_{M A X}$. The success probability for recovering $P_{r}$ increases with the increasing the value of $G_{M A X}$ and $D$.

For the estimation of the impact in the realistic environment, let us consider the situation of Fig. 2 where an attacker knows consecutive only 6 bytes of a target plaintext. Suppose that $2^{34}$ ciphertexts encrypted by randomly-chosen keys are obtained, the probability for recovering $P_{r}$ by $f_{A B S A B_{-} F}()$ with $G_{M A X}=3$ is estimated as 0.8125 . Similarly, $P_{r+1}, P_{r+2}, \ldots P_{r+5}$ are recovered by $f_{A B S A B_{-} F}()$ 
Table 2. The probabilities for recovering $P_{128}$ by using $f_{A B S A B_{-} F}()$ and $P_{128-G_{M A X}-3}$, $\ldots, P_{127}$ when $n=3072$

\begin{tabular}{lllllllll}
\hline \# of known & & & \multicolumn{6}{c}{ \# of ciphertexts } \\
\cline { 4 - 8 } plaintext bytes & $G_{M A X}$ & $D$ & $2^{31}$ & $2^{32}$ & $2^{33}$ & $2^{34}$ & $2^{35}$ & $2^{36}$ \\
\hline 3 & 0 & $2^{-32.05}$ & 0.0078 & 0.0547 & 0.0625 & 0.1250 & 0.4609 & 0.8750 \\
4 & 1 & $2^{-31.09}$ & 0.0156 & 0.0469 & 0.1797 & 0.4141 & 0.8516 & 0.9766 \\
5 & 2 & $2^{-31.55}$ & 0.0625 & 0.1484 & 0.3516 & 0.6641 & 0.9688 & 1.0000 \\
6 & 3 & $2^{-30.18}$ & 0.0703 & 0.1875 & 0.4297 & 0.8125 & 0.9922 & 1.0000 \\
7 & 4 & $2^{-29.90}$ & 0.1172 & 0.2266 & 0.5156 & 0.8750 & 0.9922 & 1.0000 \\
8 & 5 & $2^{-29.68}$ & 0.0938 & 0.2656 & 0.6250 & 0.9375 & 1.0000 & 1.0000 \\
9 & 6 & $2^{-29.50}$ & 0.1563 & 0.3438 & 0.7344 & 0.9688 & 1.0000 & $\mathrm{n} / \mathrm{a}$ \\
10 & 7 & $2^{-29.35}$ & 0.1484 & 0.3594 & 0.7656 & 0.9922 & 1.0000 & $\mathrm{n} / \mathrm{a}$ \\
11 & 8 & $2^{-29.22}$ & 0.1484 & 0.4063 & 0.7578 & 0.9922 & 1.0000 & $\mathrm{n} / \mathrm{a}$ \\
12 & 9 & $2^{-29.11}$ & 0.1484 & 0.4922 & 0.8203 & 1.0000 & 1.0000 & $\mathrm{n} / \mathrm{a}$ \\
18 & 15 & $2^{-28.66}$ & 0.2969 & 0.6172 & 0.9453 & 1.0000 & 1.0000 & $\mathrm{n} / \mathrm{a}$ \\
34 & 31 & $2^{-28.21}$ & 0.3359 & 0.7656 & 0.9766 & 1.0000 & $\mathrm{n} / \mathrm{a}$ & $\mathrm{n} / \mathrm{a}$ \\
66 & 63 & $2^{-28.03}$ & 0.3672 & 0.7656 & 0.9766 & 1.0000 & $\mathrm{n} / \mathrm{a}$ & $\mathrm{n} / \mathrm{a}$ \\
\hline
\end{tabular}

with probabilities of $0.8750,0.9375,0.9688,0.9922,0.9922$, where these parameters are $G_{M A X}=4,5,6,7,8$, respectively. Then, the attacker obtains the consecutive $12(=6+6)$ bytes with probability of $(0.8125) \cdot(0.8750) \cdot(0.9375) \cdot(0.9688)$. $(0.9922) \cdot(0.9922) \sim 0.636 . P_{r+6}, P_{r+7}, \ldots, P_{r+59}$ are expected to be recovered by $f_{A B S A B_{-} F}()$ with probability of one from Table 2 . After that, other bytes of the target plaintexts can be recovered with probability of one similar to the IOWM attack because the parameter becomes $G_{M A X}=63$. Therefore, in our attack, the only knowledge of consecutive 6 bytes of the target plaintexts enables to recover 1000 terabytes of the target plaintext with probability of about 0.636 from $2^{34}$ ciphertexts. The number of required ciphertexts of this attack is same as that of IOWM attack, while IOWM attack uses the initial 257 bytes of the keystream.

\section{Guess-and-Determine Plaintext Recovery Attack (GD Attack)}

This section gives a plaintext recovery attack which does not require any previous knowledge of the plaintext unlike the attack in Sect.3. In order to achieve it, we develop a guess-and-determine (GD) plaintext recovery method based on two strong long-term biases, the FM00 bias and the $A B S A B$ bias. Generally, in stream ciphers, the guess-and-determine method is considered as a technique for internal state recovery attacks such that a part of an internal state is determined from the other parts by exploiting the relations between the state and keystream. Our method seems to be a new class of the guess-and-determine methods for the plaintext recovery attack. 
Assuming $P_{r}$ is the target byte, the overview of our GD attack is given as follows.

1. Guess the value of $P_{r}$.

2. Recover $x$ bytes of the plaintext, $P_{r-x}, \ldots, P_{r-1}$, from $P_{r}$ (guessed in Step 1) by using the FM00 bias.

3. Recover $P_{r}^{\prime}$ from $P_{r-x}, \ldots, P_{r-1}$ (guessed in Step 2) by using the $A B S A B$ bias.

4. If $P_{r}^{\prime}$ is not equal to $P_{r}$ guessed in Step 1, the value is wrong. Otherwise the value is regarded as a candidate of correct $P_{r}$.

For each candidate of $P_{r}$, Step 1-4 are performed. The basic idea behind our GD attack is that if the value of $P_{r}$ guessed in Step 1 is correct, $P_{r}^{\prime}$ is surely same as $P_{r}$ guessed in Step 1. Two biases are used for the detection the wrong candidates of plaintext bytes.

In this section, we firstly give attack functions based on the FM00 bias for guess-and-determine methods. Then, we explain the detailed algorithm of guessand-determine methods. Finally we evaluate this attack.

\subsection{FM00 Bias for GD Attack}

The FM00 bias is a two-word bias (See Table 1), and is relatively weaker than the $A B S A B$ bias. If the simple count-up method for guessing correct plaintext byte is used, the FM00 bias is not directly used for efficient plaintext recovery attacks, because some events indexed by same $r^{*}$ are dependent each other.

For example, let us consider two events of $\left(Z_{r^{*}}, Z_{r^{*}+1}\right)=(0,0)$ and $\left(Z_{r^{*}}\right.$, $\left.Z_{r^{*}+1}\right)=(0,1)$, whose probabilities are same. Here, the relation of plaintext and ciphertext is given as $\left(P_{r}, P_{r+1}\right)=\left(C_{r} \oplus Z_{r^{*}}, C_{r+1} \oplus Z_{r^{*}+1}\right)$. If the event of $\left(Z_{r^{*}}, Z_{r^{*}+1}\right)=(0,0)$ occurs, the relation of $\left(P_{r}, P_{r+1}\right)=\left(C_{r}, C_{r+1}\right)$ hold. On the other hand, if the event of $\left(Z_{r^{*}}, Z_{r^{*}+1}\right)=(0,1)$ occurs, $\left(P_{r}, P_{r+1} \oplus\right.$ $1)=\left(C_{r}, C_{r+1}\right)$ holds. Since these probabilities are same, we can not determine whether most frequency $\left(C_{r}, C_{r+1}\right)$ is equal to $\left(P_{r}, P_{r+1}\right)$ or $\left(P_{r}, P_{r+1} \oplus 1\right)$ in the plaintext recovery attack ${ }^{3}$.

Conditional Bias Regarding the FM00 Bias. So that FM00 biases can be independently used for the plaintext recovery attack, we convert the FM00 bias into conditional bias such that $\operatorname{Pr}\left(Z_{r^{*}+1} \mid Z_{r^{*}}\right)$ (the forward) or $\operatorname{Pr}\left(Z_{r^{*}} \mid Z_{r^{*}+1}\right)$ (the backward), assuming that one byte of the plaintext can be known. Here, we consider the forward and backward conditional biases for previous two events $\left(Z_{r^{*}}, Z_{r^{*}+1}\right)=(0,0)$ and $\left(Z_{r^{*}}, Z_{r^{*}+1}\right)=(0,1)$.

The backward conditional biases, $\left(Z_{r^{*}}=0 \mid Z_{r^{*}+1}=0\right)$ and $\left(Z_{r^{*}}=0 \mid Z_{r^{*}+1}=\right.$ 1 ), are independently used for the plaintext recovery attack. Suppose that $P_{r+1}$

3 Yarrkov showed a plaintext recovery attack using the FM00 bias on his web page [15] before our results. However, the detailed description of attacks and estimations are not given, and only the source code is given. 
Algorithm 3. $f_{F M 00 \_B}()$

Require: $r, / *$ round number of plaintext to be guessed */

$P_{r+1}, / *$ known plaintext bytes */

$\left(C_{r}, C_{r+1}\right) \mathrm{s}$ of $C^{(1)}, C^{(2)}, \ldots, C^{(k)} / *$ bytes of $k$ ciphertexts encrypted by different keys */

Ensure: $P_{r}$

1: Make frequency tables of $T_{\text {count }}[$ bias $]$ of $P_{r}$ and $P_{r+1}$ for all FM00 biases regarding $P_{r+1}$ from all ciphertexts $C^{(1)}, C^{(2)}, \ldots, C^{(k)}$.

2: Convert $T_{\text {count }}[$ bias $]$ into a frequency table $T_{\text {guess }}[r]$ indexed by only $P_{r}$ with knowledge of $P_{r+1}$. Here, we only deal with the bias independent of other biases.

3: Regard most frequency one in table $T_{\text {guess }}[r]$ as the correct $P_{r}$.

4: Output $P_{r}$

is obtained, the values of $Z_{r^{*}+1}=0$ is computed by $Z_{r^{*}+1}=C_{r+1} \oplus P_{r+1}$. Then, two tables of $Z_{r^{*}}=0$ are obtained from two backward conditional biases $\left(Z_{r^{*}}=\right.$ $\left.0 \mid Z_{r^{*}+1}=0\right)$ and $\left(Z_{r^{*}}=0 \mid Z_{r^{*}+1}=1\right)$. In these tables, it is expected that most frequency values of these tables indicate same value of the plaintext, because source event $Z_{r^{*}+1}$ are different. Thus, these frequency tables are efficiently merged to recover the plaintext byte $P_{r}$.

On the other hand, the forward conditional biases $\left(Z_{r^{*}+1}=0 \mid Z_{r^{*}}=0\right)$ and $\left(Z_{r^{*}+1}=1 \mid Z_{r^{*}}=0\right)$ are not independently used. Even if two tables of $Z_{r^{*}}=0$ obtained from $\left(Z_{r^{*}+1}=0 \mid Z_{r^{*}}=0\right)$ and $\left(Z_{r^{*}+1}=1 \mid Z_{r^{*}}=0\right)$ are merged, it is expected that two peaks of $Z_{r^{*}+1}=0$ and $Z_{r^{*}+1}=1$ are observed due to same source event $Z_{r^{*}}=0$.

Therefore, if the source events of conditional bias (the forward is $Z_{r^{*}}$ and the backward is $Z_{r^{*}+1}$ ) are different, these events can be independently used. Note that events for positive bias and negative bias are not dependent even if the source events of conditional bias are same.

Attack Functions Based on the FM00 Bias. By using all the independent conditional biases, we construct the forward and backward functions for the guess-and-determine attack based on the FM00 bias as follows:

$f_{F M 00_{-} F}()$ : Find an unknown byte $P_{r}$ from pre-known a byte of a plaintext $P_{r-1}$.

$f_{F M 00_{B} B}()$ : Find an unknown byte $P_{r}$ from pre-known a byte of a plaintext $P_{r+1}($ See Algorithm 3).

The algorithm of $f_{F M 00_{-} F}()$ is given by replacing "+" of subscripts of variables in Algorithm 3 to "-". Figure 3 illustrates the procedures of $f_{F M 00_{-} F}()$ and $f_{F M 00_{-} B}()$.

The number of independent events of the forward conditional bias $N_{f}$ and that of the backward conditional bias $N_{b}$ in each index $i$ are shown in Table 3 . When index $i=0$, the all events of backward conditional bias are independent, and $N_{b}=5$. On the other hand, two events of forward conditional bias $Z_{r^{*}+1}=$ $1 \mid Z_{r^{*}}=N-1$ and $Z_{r^{*}+1}=2 \mid Z_{r^{*}}=N-1$ are not independent, and $N_{f}=$ 


$$
\begin{aligned}
& P_{r-1} P_{r} \\
& P_{r} P_{r+1}
\end{aligned}
$$

Fig. 3. The guess-and-determine methods based on the conditional bias of the FM00 bias

$5-2=3$. For all index $i, N_{b}$ is larger than $N_{f}$. Hence, the success probability of $f_{F M 00_{-} B}()$ is larger than that of $f_{F M 00_{-} F}()$.

\subsection{Plaintext Recovery Method for Recovering Any Plaintext Byte}

Our GD attack utilizes the backward conditional bias of the FM00 bias and the forward $A B S A B$ bias.

To begin with, a plaintext byte $P_{r}$ is guessed from $N$ candidates. Then, our attack sequentially recovers $P_{r-1} \rightarrow P_{r-2} \rightarrow \ldots$ from $P_{r}$ and the ciphertexts by using $f_{F M 00_{-} B}()$ in the backward manner. Since the number of the candidates of $P_{x}$ is $N$, the number of candidates of $\left(P_{r}, P_{r-1}, P_{r-2}, \ldots\right)$ is also $N$. In order to detect the wrong candidates of $P_{r}$, we use $f_{A B S A B_{-} F}()$, which is based on the other bias. In particular, $P_{r}^{\prime}$ is obtained from ciphertexts and the candidate of plaintext bytes $\left(P_{r-1}, P_{r-2}, \ldots\right)$ by using $f_{A B S A B_{-} F}()$ with $G_{M A X}$. If the number of ciphertexts is enough larger and $P_{r}$ is correctly guessed, the relation of $P_{r}=P_{r}^{\prime}$ surely holds. Otherwise the probability that $P_{r}=P_{r}^{\prime}$ holds is $1 / N$. After this method, about two candidates of $P_{r}$ are expected to be left. If the number of the candidates of $P_{r}$ is not one, the same method is repeated for $P_{r-1}^{\prime}, P_{r-2}^{\prime}, \ldots$, which are obtained by $P_{r}$. If $P_{r}$ is correct, these method correctly works. In most cases, the number of repeating this method $N_{\text {repeat }}$ is less than three. Figure 4 shows the procedure of our plaintext recovery attack for recovering any plaintext byte. The detail of our attack for recovering any plaintext byte $P_{r}$ is given in Algorithm 4.

We consider the parameter $G_{M A X}$ for the $A B S A B$ bias. It should be chosen so that $A B S A B$ bias is stronger than the FM00 bias to efficiently detect wrong candidates. From Lemma 2, given $1 / D$ samples, $\operatorname{Pr}_{\text {distinguish }}$ become constant. Since the probability of the plaintext recovery attack depends on $\operatorname{Pr}_{\text {distinguish }}$, we evaluate our attack by the number of required ciphertexts for obtaining $1 / D$ samples. For example, $D$ of the backward conditional bias of the FM00 bias is estimated as $D=N^{-3}=2^{-24}$ for $N=256$ and $i=3,4, \ldots, N-4$. From Table 3, there are seven independent events of the FM00 conditional biases in this case. As mentioned before, these biases are independently used. Thus, the probability that a ciphertext matches one of these source events is $7 / N$. The number of the required ciphertexts is $(N / 7) \cdot(1 / D)=2^{29.19}$ for $1 / D$ samples. On the other hand, discriminations $D$ of the $A B S A B$ bias and these number of 
Table 3. Events of the conditional bias of the FM00 bias in each index $i\left(=r^{*} \bmod N\right)$

\begin{tabular}{|c|c|c|c|c|c|}
\hline Index $i$ & $Z_{r^{*}}$ & $Z_{r^{*}+1}$ & Conditional probability & $N_{f}$ & $N_{b}$ \\
\hline \multirow{5}{*}{0} & 0 & 0 & $N^{-1} \cdot\left(1+N^{-1}\right)$ & \multirow{5}{*}{3} & \multirow{5}{*}{5} \\
\hline & 1 & $N-1$ & $N^{-1} \cdot\left(1+N^{-1}\right)$ & & \\
\hline & $N-1$ & 1 & $N^{-1} \cdot\left(1+N^{-1}\right)$ & & \\
\hline & $N-1$ & 2 & $N^{-1} \cdot\left(1+N^{-1}\right)$ & & \\
\hline & $N-1$ & $N-1$ & $N^{-1} \cdot\left(1-N^{-1}\right)$ & & \\
\hline \multirow{6}{*}{1} & 0 & 0 & $N^{-1} \cdot\left(1+2 \cdot N^{-1}\right)$ & \multirow{6}{*}{4} & \multirow{6}{*}{6} \\
\hline & 2 & $N-1$ & $N^{-1} \cdot\left(1+N^{-1}\right)$ & & \\
\hline & $N-1$ & 3 & $N^{-1} \cdot\left(1+N^{-1}\right)$ & & \\
\hline & $N-1$ & 2 & $N^{-1} \cdot\left(1+N^{-1}\right)$ & & \\
\hline & $N-1$ & $N-1$ & $N^{-1} \cdot\left(1-N^{-1}\right)$ & & \\
\hline & 0 & 2 & $N^{-1} \cdot\left(1-N^{-1}\right)$ & & \\
\hline \multirow{8}{*}{2} & 0 & 0 & $N^{-1} \cdot\left(1+N^{-1}\right)$ & \multirow{8}{*}{4} & \multirow{8}{*}{8} \\
\hline & 0 & 1 & $N^{-1} \cdot\left(1+N^{-1}\right)$ & & \\
\hline & 3 & $N-1$ & $N^{-1} \cdot\left(1+N^{-1}\right)$ & & \\
\hline & $N-1$ & 3 & $N^{-1} \cdot\left(1+N^{-1}\right)$ & & \\
\hline & $N-1$ & 4 & $N^{-1} \cdot\left(1+N^{-1}\right)$ & & \\
\hline & $N / 2+1$ & $N / 2+1$ & $N^{-1} \cdot\left(1+N^{-1}\right)$ & & \\
\hline & $N-1$ & $N-1$ & $N^{-1} \cdot\left(1-N^{-1}\right)$ & & \\
\hline & 0 & 3 & $N^{-1} \cdot\left(1-N^{-1}\right)$ & & \\
\hline \multirow{7}{*}{$3,4, \ldots, N-4$} & 0 & 0 & $N^{-1} \cdot\left(1+N^{-1}\right)$ & \multirow{7}{*}{3} & \multirow{7}{*}{7} \\
\hline & 0 & 1 & $N^{-1} \cdot\left(1+N^{-1}\right)$ & & \\
\hline & $i+1$ & $N-1$ & $N^{-1} \cdot\left(1+N^{-1}\right)$ & & \\
\hline & $N-1$ & $i+1$ & $N^{-1} \cdot\left(1+N^{-1}\right)$ & & \\
\hline & $N-1$ & $i+2$ & $N^{-1} \cdot\left(1+N^{-1}\right)$ & & \\
\hline & $N-1$ & $N-1$ & $N^{-1} \cdot\left(1-N^{-1}\right)$ & & \\
\hline & 0 & $i+1$ & $N^{-1} \cdot\left(1-N^{-1}\right)$ & & \\
\hline \multirow{6}{*}{$N-3$} & 0 & 0 & $N^{-1} \cdot\left(1+N^{-1}\right)$ & \multirow{6}{*}{4} & \multirow{6}{*}{6} \\
\hline & 0 & 1 & $N^{-1} \cdot\left(1+N^{-1}\right)$ & & \\
\hline & $N-2$ & $N-1$ & $N^{-1} \cdot\left(1+N^{-1}\right)$ & & \\
\hline & $N-1$ & $N-2$ & $N^{-1} \cdot\left(1+N^{-1}\right)$ & & \\
\hline & $N-1$ & $N-1$ & $N^{-1} \cdot\left(1-N^{-1}\right)$ & & \\
\hline & 0 & $N-2$ & $N^{-1} \cdot\left(1-N^{-1}\right)$ & & \\
\hline \multirow{4}{*}{$N-2$} & 0 & 0 & $N^{-1} \cdot\left(1+N^{-1}\right)$ & \multirow{4}{*}{2} & \multirow{4}{*}{2} \\
\hline & 0 & 1 & $N^{-1} \cdot\left(1+N^{-1}\right)$ & & \\
\hline & $N-1$ & 0 & $N^{-1} \cdot\left(1+N^{-1}\right)$ & & \\
\hline & 0 & $N-1$ & $N^{-1} \cdot\left(1-N^{-1}\right)$ & & \\
\hline \multirow{5}{*}{$N-1$} & 0 & 1 & $N^{-1} \cdot\left(1+N^{-1}\right)$ & \multirow{5}{*}{1} & \multirow{5}{*}{3} \\
\hline & 0 & $N-1$ & $N^{-1} \cdot\left(1+N^{-1}\right)$ & & \\
\hline & $N-1$ & 0 & $N^{-1} \cdot\left(1+N^{-1}\right)$ & & \\
\hline & $N-1$ & 1 & $N^{-1} \cdot\left(1+N^{-1}\right)$ & & \\
\hline & $N-1$ & $N-1$ & $N^{-1} \cdot\left(1-N^{-1}\right)$ & & \\
\hline
\end{tabular}




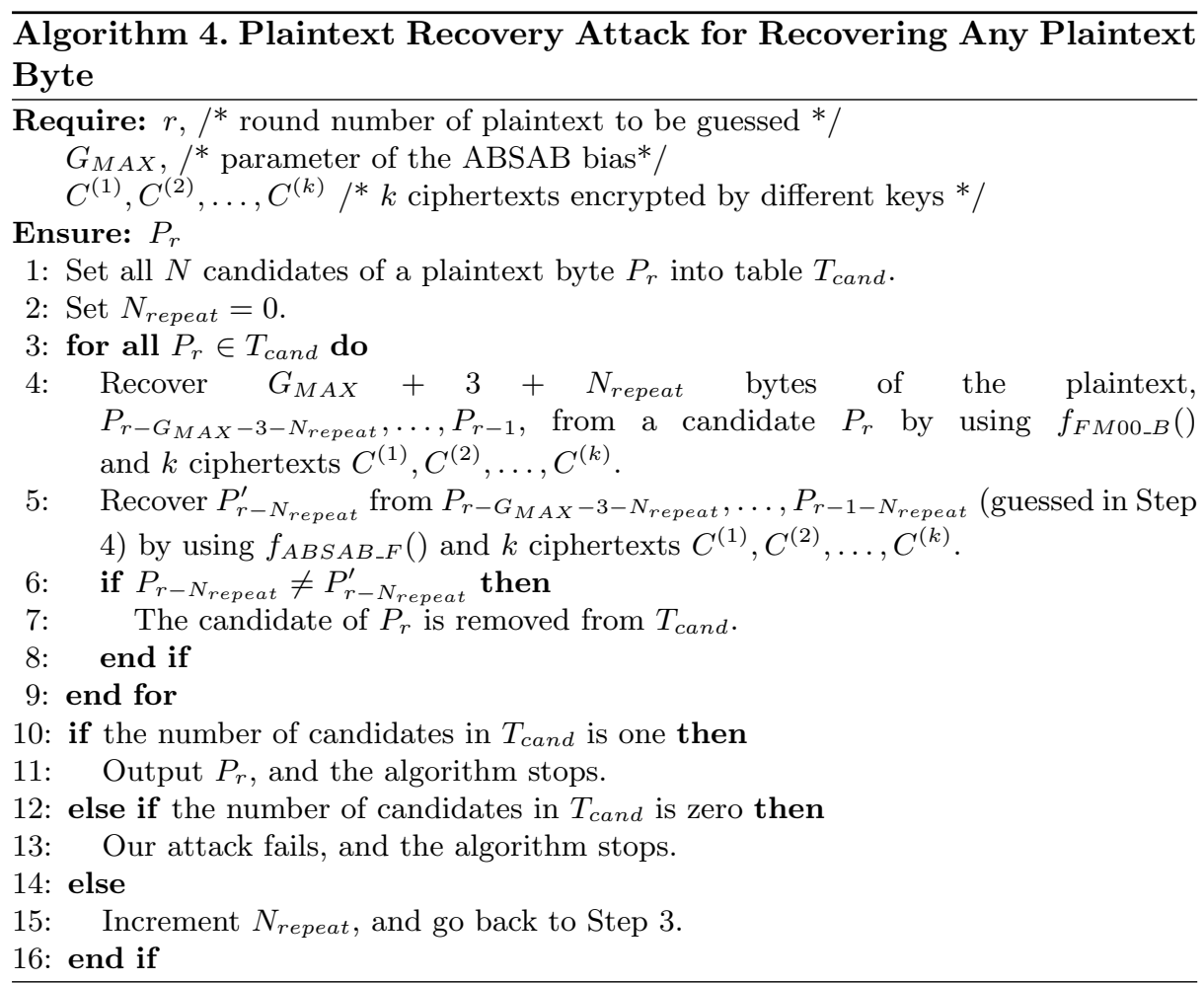

required ciphertexts for recovering a plaintext byte are shown as $\left(D=2^{-29.22}\right.$, $1 / D=2^{29.22}$ ciphertexts) for $G_{M A X}=8$ and $\left(D=2^{-29.11}, 1 / D=2^{29.11}\right.$ ciphertexts) for $G_{M A X}=9$. Therefore $G_{M A X}=9$ is chosen for $N=256$ and $i=3,4, \ldots, N-4$.

\subsection{Experimental Results}

We perform the computer experiment for demonstrating the effectiveness of our attack with $G_{M A X}$ on $\mathrm{RC} 4-\operatorname{drop}(n)$ in the broadcast setting when $N=256$ and $n=3072$. In this experiment, $P_{128}$ is recovered from ciphertexts without the knowledge of the target plaintext. The parameters of the backward conditional bias of the FM00 bias, index $i$, satisfy $r^{*} \bmod 256=i \in\{3,4, \ldots, N-4\}$. Hence, $G_{M A X}=9$ is used as the parameter of the $A B S A B$ bias.

First, in order to evaluate $f_{F M 00_{-} B}()$, we obtain the success probability for recovering $P_{114}, \ldots, P_{127}$ under the condition that the correct $P_{128}$ is given. The success probabilities when $2^{29}$ to $2^{35}$ ciphertexts are given is shown in Table 4 , where the number of tests is 256 . The experimental result shows that all bytes of $P_{114}, \ldots, P_{127}$ are recovered from $2^{35}$ ciphertexts encrypted by randomlychosen different keys with probability of one by using $f_{F M 00_{B} B}()$. This results also shows that if one byte of the plaintext is known, $2^{33}$ ciphertexts enable to 


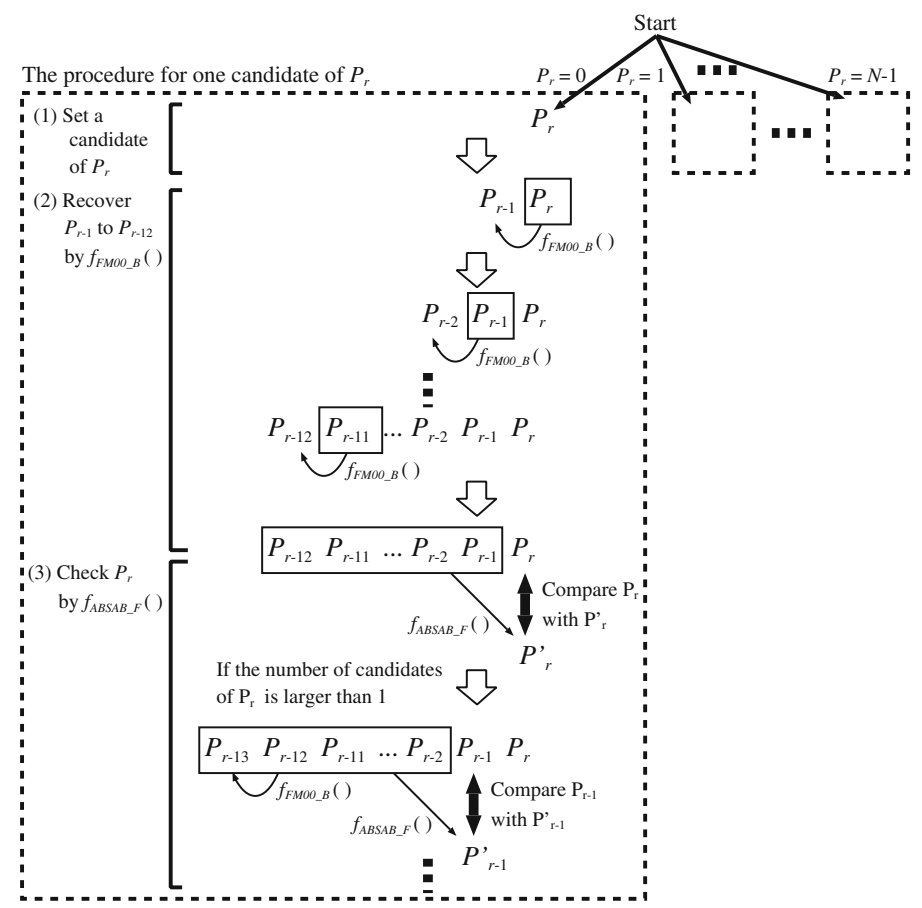

Fig. 4. The procedure of our plaintext recovery attack for recovering any plaintext byte $\left(G_{M A X}=9\right)$

recover the other one byte with probability of about 0.8 . Interestingly, it is more efficient than the attack in Sect. 3 when $G_{M A X}$ is small. The attack in Sect. 3 are improved by using $f_{F M 00_{-} B}()$. If consecutive 6 bytes of the plaintext are known, the other bytes can be recovered from $2^{34}$ with probability of about 0.984 , while that of the attack in Sect. 3 is about 0.636 .

Then, we estimate the success probability for recovering $P_{128}$ from only ciphertexts by our plaintext recovery attack. The success probability when $2^{32}$ to $2^{35}$ ciphertexts are given is shown in Table 5 , where the number of tests is 256. This experiment requires about one week with one CPU core (Intel(R) Core(TM) i7 CPU 920@ $2.67 \mathrm{GHz}$ ) to obtain the result of one plaintext. The experimental result shows that our attack can recover the target plaintext byte $P_{128}$ with probability of one from $2^{35}$ ciphertexts encrypted by randomly-chosen different keys. Remaining plaintext bytes, namely $P_{r}(r \neq 128)$ can be recovered by repeating our attack or using our attack functions $f_{A B S A B_{-} F}(), f_{A B S A B_{-} B}()$, $f_{F M 00_{-} F}()$, and $f_{F M 00_{-} B}()$. Especially, in the cases of $i=N-2, N-1$, the success probabilities of conditional bias of the FM00 bias are relatively small than that of others cases. These bytes should be recovered by using $f_{A B S A B_{-} F}()$, $f_{A B S A B_{-} B}()$ for an efficient recovery attack after other bytes are recovered by using our GD attack. 
Table 4. Success probabilities of $f_{F M 00_{-} B}()$ for recovering $\left(P_{114}, \ldots, P_{127}\right)$ under the condition that the correct $P_{128}$ is given when $n=3072$

\begin{tabular}{llllllll}
\hline & \multicolumn{7}{c}{ \# of ciphertexts } \\
\cline { 2 - 8 } & $2^{29}$ & $2^{30}$ & $2^{31}$ & $2^{32}$ & $2^{33}$ & $2^{34}$ & $2^{35}$ \\
\hline$P_{114}$ & 0.0039 & 0.0039 & 0.0039 & 0.0078 & 0.0781 & 0.8750 & 1.0000 \\
$P_{115}$ & 0.0039 & 0.0039 & 0.0078 & 0.0117 & 0.1055 & 0.8828 & 1.0000 \\
$P_{116}$ & 0.0078 & 0.0000 & 0.0078 & 0.0117 & 0.1133 & 0.8828 & 1.0000 \\
$P_{117}$ & 0.0039 & 0.0039 & 0.0078 & 0.0078 & 0.1328 & 0.8945 & 1.0000 \\
$P_{118}$ & 0.0078 & 0.0195 & 0.0078 & 0.0078 & 0.1758 & 0.9023 & 1.0000 \\
$P_{119}$ & 0.0078 & 0.0000 & 0.0078 & 0.0117 & 0.1992 & 0.9180 & 1.0000 \\
$P_{120}$ & 0.0039 & 0.0039 & 0.0078 & 0.0156 & 0.2422 & 0.9258 & 1.0000 \\
$P_{121}$ & 0.0039 & 0.0039 & 0.0117 & 0.0078 & 0.2773 & 0.9492 & 1.0000 \\
$P_{122}$ & 0.0039 & 0.0078 & 0.0039 & 0.0117 & 0.3203 & 0.9570 & 1.0000 \\
$P_{123}$ & 0.0000 & 0.0117 & 0.0117 & 0.0195 & 0.3672 & 0.9688 & 1.0000 \\
$P_{124}$ & 0.0078 & 0.0039 & 0.0195 & 0.0391 & 0.4727 & 0.9844 & 1.0000 \\
$P_{125}$ & 0.0078 & 0.0039 & 0.0078 & 0.0742 & 0.5820 & 0.9883 & 1.0000 \\
$P_{126}$ & 0.0039 & 0.0078 & 0.0391 & 0.1602 & 0.6680 & 0.9922 & 1.0000 \\
$P_{127}$ & 0.0430 & 0.0898 & 0.1719 & 0.3984 & 0.8008 & 0.9922 & 1.0000 \\
\hline
\end{tabular}

Table 5. Success probabilities of our attack for recovering $P_{128}$ when $n=3072$

\begin{tabular}{ccccc}
\hline & \multicolumn{4}{c}{ \# of ciphertexts } \\
\cline { 2 - 5 } $2^{32}$ & $2^{33}$ & $2^{34}$ & $2^{35}$ \\
\hline$P_{128}$ & 0.0039 & 0.1133 & 0.9102 & 1.0000 \\
\hline
\end{tabular}

Given $2^{34}$ ciphertexts encrypted by randomly-chosen different keys, our attack can recover any plaintext byte with probability of about 0.91 . The number of required ciphertexts are same as that of IOWM attack on original RC4, which does not discard initial keystream bytes. In addition, even if $2^{33}$ ciphertexts are given, our attack is more efficient than a random guess.

Also, this attack is applicable to original RC4 with constant success probability regardless of the position of plaintext bytes, while that of the IOWM attack decrease for the later plaintext byte. It is an advantage of our attack from the IOWM attack on the original RC4.

\section{Conclusion}

In this paper, we have evaluated the security of relatively secure RC4 implementation called RC4-drop $(n)$, which discards the first $n$ bytes of the keystream. We proposed two advanced plaintext recovery attacks that can recover any byte of a plaintext on $\mathrm{RC} 4-\operatorname{drop}(n)$ in the broadcast setting or the multi-session setting. The first attack is the modified IOWM attack. Using partial knowledge of the target plaintext, the other bytes can be recovered from ciphertexts encrypted by different keys. The attack can recover 1000 terabytes of a target plaintext 
with the high probability from $2^{34}$ ciphertexts encrypted by different keys if the knowledge of only consecutive 6 bytes of the target plaintext is given. The second attack does not rely on any previous knowledge of a plaintext. In order to achieve it, we developed a guess-and-determine plaintext recovery method based two strong long-term biases. Given $2^{35}$ ciphertext encrypted by different keys, any byte of a plaintext can be recovered with probability close to one from only ciphertexts. The amount of ciphertext is almost same as the IOWM attack on original $\mathrm{RC} 4$. Therefore, $\mathrm{RC} 4$ is not secure even if the enough initial keystream bytes are disregarded.

We recommend to replace RC4 with other stream ciphers [8] or the algorithms of authenticated encryption in the practical protocols and software applications.

A future work is to compare our attack with the method of $[1,2]$ in the same conditions. In addition, we will combine our attack with the method of $[1,2]$ for obtaining more efficient attacks.

Acknowledgments. This work was supported in part by Grant-in-Aid for Scientific Research (C) (KAKENHI 23560455) and Grant-in-Aid for Young Scientists (B) (KAKENHI 25730085) for Japan Society for the Promotion of Science.

\section{References}

1. AlFardan, N.J., Bernstein, D.J., Paterson, K.G., Poettering, B., Schuldt, J.C.N.: On the security of RC4 in TLS. In: USENIX Security 2013 (2013) (to appear)

2. AlFardan, N.J., Bernstein, D.J., Paterson, K.G., Poettering, B., Schuldt, J.C.N.: On the security of RC4 in TLS and WPA. http://www.isg.rhul.ac.uk/tls/ RC4biases.pdf (2013)

3. Canvel, B., Hiltgen, A.P., Vaudenay, S., Vuagnoux, M.: Password interception in a SSL/TLS channel. In: Boneh, D. (ed.) CRYPTO 2003. LNCS, vol. 2729, pp. 583-599. Springer, Heidelberg (2003)

4. Fluhrer, S.R., McGrew, D.A.: Statistical analysis of the alleged RC4 keystream generator. In: Schneier [14], pp. 19-30

5. Sen Gupta, S., Maitra, S., Paul, G., Sarkar, S.: Proof of empirical RC4 biases and new key correlations. In: Miri, A., Vaudenay, S. (eds.) SAC 2011. LNCS, vol. 7118, pp. 151-168. Springer, Heidelberg (2012)

6. Sen Gupta, S., Maitra, S., Paul, G., Sarkar, S.: (Non-)random sequences from (Non-) random permutations - analysis of RC4 stream cipher. J. Cryptol. 1-42 (2012). http://dblp.uni-trier.de/rec/bibtex/journals/joc/GuptaMPS14

7. Isobe, T., Ohigashi, T., Watanabe, Y., Morii, M.: Full plaintext recovery attack on broadcast RC4. Preproceeding of Fast Software Encryption (FSE) (2013)

8. Josefsson, S., Strombergson, J., Mavrogiannopoulos, N.: The salsa20 stream cipher for transport layer security (TLS) and datagram transport layer security (DTLS). Network Working Group Internet-Draft, March 2013. http://tools.ietf.org/html/ draft-josefsson-salsa20-tls-01 (2013)

9. Maitra, S., Paul, G., Sen Gupta, S.: Attack on broadcast RC4 revisited. In: Joux, A. (ed.) FSE 2011. LNCS, vol. 6733, pp. 199-217. Springer, Heidelberg (2011)

10. Mantin, I.: Analysis of the stream cipher RC4. Master's Thesis, The Weizmann Institute of Science, Israel. http://www.wisdom.weizmann.ac.il/itsik/RC4/ rc4.html (2001) 
11. Mantin, I.: Predicting and distinguishing attacks on RC4 keystream generator. In: Cramer, R. (ed.) EUROCRYPT 2005. LNCS, vol. 3494, pp. 491-506. Springer, Heidelberg (2005)

12. Mantin, I., Shamir, A.: A practical attack on broadcast RC4. In: Matsui, M. (ed.) FSE 2001. LNCS, vol. 2355, pp. 152-164. Springer, Heidelberg (2002)

13. Mironov, I.: (not so) random shuffles of RC4. In: Yung, M. (ed.) CRYPTO 2002. LNCS, vol. 2442, pp. 304-319. Springer, Heidelberg (2002)

14. Schneier, B. (ed.): FSE 2000. LNCS, vol. 1978. Springer, Heidelberg (2001)

15. Yarrkov, E.: Why the recent RC4 attack doesn't surprise me. https://cipherdev. org/rc4_2013-03-13.html (2013) 